
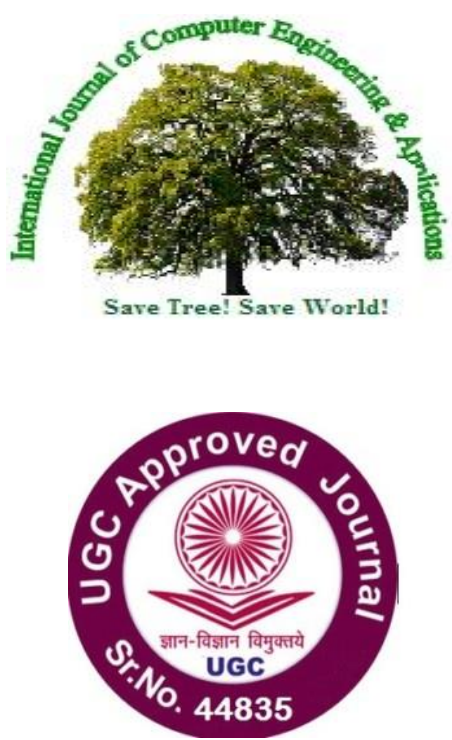

\title{
A STUDY ON ROLE OF ARTIFICIAL INTELLIGENCE, ROBOTICS AND MACHINE LEARNING IN SALES AND MARKETING AREA
}

\author{
Reshma Motwani ${ }^{1}$, Mayuri Patel ${ }^{2}$, Dr. Shabana Memon ${ }^{3}$, Prof. Nripesh Nrip ${ }^{4}$ \\ ${ }^{1 \& 2}$ Student, ${ }^{3 \& 4}$ Assistant Professor, Bharati Vidyapeeth (Deemed to be University) Pune \\ Institute of Management Kolhapur Maharashtra India
}

\begin{abstract}
:
The use of Personal Digital Assistants (Smart Phones/ Tablets) is rapidly increasing day by day and so does the use of other social media applications. People tend to buy products online from different social media applications such as Flipkart, Amazon, Jio Mart, etc. So, we can say the market is global now, any kind of product can be purchased online today. This creates a competition among competitors to increase their sales and improve their marketing strategies constantly. This article shows a limelight on how techniques of Artificial Intelligence, Robotics and Machine Learning create a significant impact on sales and marketing process.
\end{abstract}

Keywords: Artificial Intelligence, Robotics, Machine Learning, Marketing Automation.

\section{[1] INTRODUCTION}

The term Artificial Intelligence was first coined by John McCarthy in 1956 when he held the first academic conference on the subject. In the late 1990's as the computational power increased and with an explosion of data, the AI revival sparked which still has continued to present times.

Today, AI can be incorporated in various different types of technologies such as Automation, Machine Learning, Machine Vision, Natural language processing, Robotics, etc.

Machine learning is considered to be a part of Artificial Intelligence. It is the science of getting 
a computer to act without programming. It is the study of computer algorithms that can improve automatically through experience and by the use of data [1]. Whereas, Robotics is considered as the field of engineering which focuses on the design and manufacturing of robots. Robots are often used to perform tasks that are difficult for humans to perform or perform consistently [2].

While integrating Artificial Intelligence with different technologies, AI has changed the world of business dynamically. One of the most significant applications of AI is in the sales and marketing field. The present research is aimed to find out the impact of artificial intelligence, machine learning and robotics on the business world specially in context to the field of sales and marketing.

\section{[2] LITERATURE REVIEW AND THEORETICAL FRAMEWORKS}

\section{A) ARTIFICIAL INTELLIGENCE}

Artificial Intelligence has been studied for decades and yet is still one of the most enthralling subjects in the field of computer science. AI was first founded as an academic discipline in 1956 by John McCarthy. It has faced several waves of optimism which were followed by the disappointment and loss of funding which is known as 'AI Winter' and again followed by new approaches, success and renewed funding.

According to the father of AI, John McCarthy, Artificial Intelligence is "The science and engineering of making intelligent machines [3]."

AI includes sub-categories such as Machine learning, deep learning, natural language processing, expert system, robotics, machine vision, speech recognition which manufacture real world applications of AI including search suggestions, voice recognitions, virtual assistants, image recognition, etc. AI can be categorized as either weak AI or strong AI.

- Weak AI, also known as narrow AI, is an AI system that is designed and trained to complete a specific task [2].

- $\quad$ Strong AI, also known as artificial general intelligence (AGI), describes programming that can replicate the cognitive abilities of the human brain. When presented with an unfamiliar task, Strong AI can use fuzzy logic, to apply knowledge from one domain to another and find a solution autonomously. In theory, a strong AI should be able to pass both a Turing Test and the Chinese Room Test [2].

\section{B) AI IN SALES AND MARKETING}

The progressions made in $\mathrm{AI}$ and its executions in various domains have resulted in the advancement of AI technologies. The Traditional techniques of sales and marketing have been replaced by AI techniques and will continue to do so as the techniques in Artificial Intelligence keeps on developing.

Today, AI is used to support marketing managers in various tasks and operations including digital marketing (buying), external email marketing, lead generation, web development SEO, social media monitoring and A/B testing, etc.

AI in the field of marketing is not only used to attract new customers towards your product but also engage the existing customers in returning towards your organization. AI can increase the reach that is attract visitors with a range of various inbound techniques such as AI generated content, Smart 
content curation, voice search, programmatic media buying, propensity modeling, and much more.

Machine Learning techniques such as sentiment analysis and reputation scoring, customer personalization, lead scoring, customer segmentation and discovery, recommender systems, chatbots and virtual assistants, marketing mix optimization and other such techniques have made clear that every aspect of marketing can and will be improved with the help of machine learning.

A simple use of robots in sales and marketing could be that it can be used to aggregate contacts for sales staff so that your sales reps do not have to spend time searching for companies, names, phone numbers, and other critical contact information. Then, bots can be used to update your CRM with this new contact data [4].

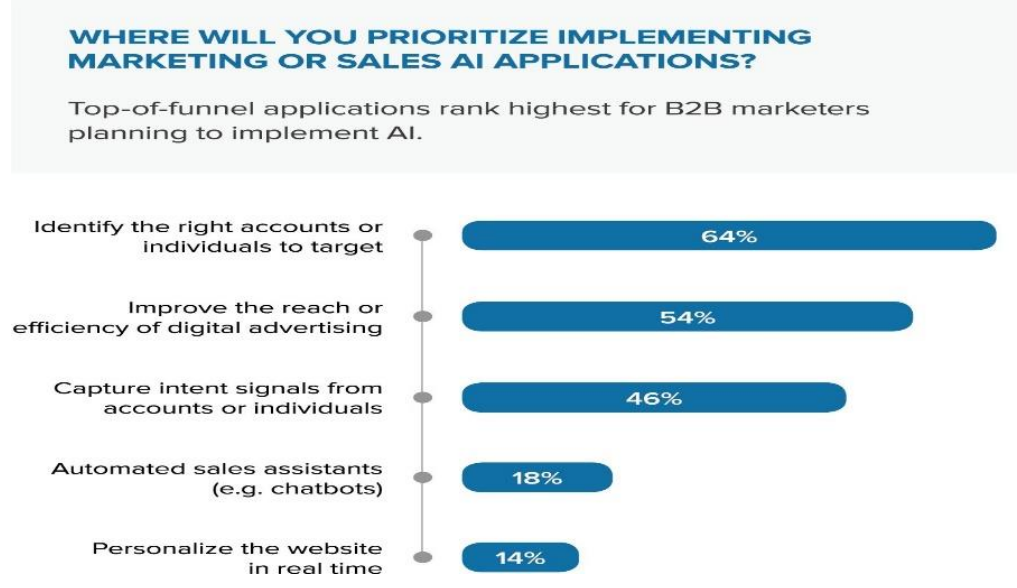

(Source- Demandbase, Apr 03, 2019- https://www.prnewswire.com/news-releases/85-of-marketers-usingartificial-intelligence-believe-it-will-drive-double-digit-revenue-growth-within-two-years-300823481.html

In terms of priorities for AI investments, study participants planning their AI implementations list top-of funnel applications as priorities:

1. Identify the right accounts or individuals to target (64\%)

2. Improve the reach or efficiency of digital advertising (54\%)

3. Capture intent signals from accounts or individuals (46\%)

\section{[3] TECHNIQUES OF AI, ROBOTICS AND MACHINE LEARNING USED IN THE FIELD OF SALES AND MARKETING}

The primary goal of any business is to maximize the profit. So far we have come across different applications of AI, for instance, AI-powered computer vision systems in self-driving cars are able to identify obstacles just like people do allowing machines to take control over the wheel, voice assistants like Alexa or Siri understands your words just like another person and then responds in kind all using AI, AI recommendation engines are used by Amazon and Netflix to offer up products and movies you might like by making assumptions about your preferences like a movie enthusiast would do.

AI describes many different types of smart technologies which impact sales and marketing performance in profound ways.

\section{A) AI AND MACHINE LEARNING IN SALES:}

It is evident that Artificial Intelligence has boosted the sales process. AI in sales is used to 
analyze large datasets. It uses advanced computer science techniques to extract insights from data. These insights can then be used to make predictions, recommendations and decisions.

Machine Learning is a subset of AI that identifies patterns based on large sets of data and use these patterns to make predictions. Then, it uses more and more data to improve those predictions over time. Moreover, Technology powered by Machine Learning gets better over time, often without human involvement [6].

\section{PREDICTIVE FORECASTING}

Predictive Forecasting refers to AI systems that can predict or forecast outcomes using historical data to inform future results. Common predictions that AI sales systems can make are:

- Deals or Prospects likely to close

- Deals or prospects to target next

- $\quad$ New customers that may be interested in your selling products

The accuracy of these predictions depends upon the quality of data, inputs given and the system being used. Given the right inputs in the past and in the present, AI is capable to show you who is most likely to buy next. Such Predictive Forecasting can also create value for sales teams internally. Using the same type of data analysis, AI can help sales manager to forecast their team performance for the quarter well in advance so that they can take the immediate steps required.

\section{LEAD SCORING AND PRIORITIZATION}

While Artificial Intelligence can look dispassionately at large datasets from a number of sources and tells you which leads you should prioritize based on the score AI has given them, Humans make such decisions based on gut instinct and incomplete information. The AI algorithm can compile historical information about a client, along with social media postings and the salesperson's customer interaction history (i.e., emails sent, voicemails left, text messages sent, etc.) and rank the opportunities or leads in the pipeline according to their chances of closing successfully. In this case, humans can bring level of logic and standardization to the process.

\section{EXPERT RECOMMENDATIONS}

Apart from predictions and prioritizations, some AI systems may actually help to recommend sales actions, going so far to tell the sales teams which actions the system thinks makes the most sense based on your goals and insights from your data. These systems may include recommendations that may include advice on how to price a deal, who should be targeted next, which customers to target first with upsells or cross-sells. The result is targeted guidance on what actions must the salespeople put focus on and can free up bandwidth to close deals, rather than deliberating about what to do next.

\section{PERFROMANCE AND PRODUCTIVITY ENHANCEMENT}

With the help of AI, we can also automate and augment work to take away some of the drudgery that distracts you from the higher-value tasks. It can help with everything from managing your calendar to scheduling meetings to accessing a sales team's pipeline by automatically doing things for you or making them easier by using your historical usage data to make decisions.

\section{B) AI AND MACHINE LEARNING IN MARKETING:}

It is clear that AI plays a vital role in helping marketing professionals connect to their consumers. 
While implementing AI in marketing process key factors such as establishing goals, data privacy standards, data quantity and sources, maintaining data quality, selecting an AI platform should be taken into consideration. Some Techniques of AI and Machine Learning used in marketing are described below [7][8].

\section{BIDDING ON PROGRAMMATIC MEDIA BuYs}

Earlier, the marketing teams made decisions about where to place the advertisements and messaging and create an informed plan based on user preferences. However, these marketing teams are not flexible to alter the plan in real time based on latest consumer information. AI is used by marketing professionals to overcome this challenge through programmatic advertising. The programmatic platforms leverage machine learning to bid on ad space relevant to target audiences in real time. The data such as interests, location, purchase history, buyer intent and more helps in informing the bid which enables marketing teams to target the right channels at the right time for competitive pricing. This increases marketing flexibility to meet customers as their need and interests evolve.

\section{CONTENT CURATIONS AND RECOMMENDATIONS}

We all are familiar with the now-commonplace "If you liked this, you might also like.." or "top pins that you might like.." content recommendations at the end of many articles on web and notifications from different applications. These recommendations are actually based on deep learning and other AI, where machines analyze large chunks of data about people's behavior on the web to determine what they are most likely to want to do next.

\section{WRITING SEO OPTIMIZED HEADLINES}

Deep learning and neural networks are used to teach machines to write headlines that have a direct impact on the reader and would lure the user to click on the link immediately.

\section{GRANULAR PERSONALIZATION}

A highly granular level of personalization is expected by today's consumers. Marketing messages should be informed by a user's interests, purchase history, location and a host of other data points. AI helps marketing teams to learn about consumer preferences on individual level. This helps brands to create curated experiences based on customers unique tastes. Major ecommerce websites use AI to recommend products based on the products you click and buy, Spotify uses AI to create customized playlists for its consumers based on the prior listening, current hits across genres and what kind of music is being talked about.

\section{SPEECH RECOGNITION}

Speech recognition is becoming increasingly important in the reals of SEO and content marketing, since the rise in voice search content must be optimized for queries that come in question form, rather than simple keywords.

\section{AD TARGETING}

Sites like Google and Facebook use bits of code to track website visitors and target ads accordingly. Most marketers agree that the process is clunky and difficult to use. Google is now beginning to experiment with a new AI technology called recurrent neural networks (RNN) that is capable of "remembering" bits of information for short periods of time, potentially eliminating the need for specialized ad targeting code in the near future. 


\section{7.СНАТвотS}

As the marketing channels expand, marketers need a way to manage those interactions cost effectively. Many websites and marketers use chatbots or other AI that are able to interact with humans to answer routine questions, assist with purchases, solve queries and much more.

\section{MARKETING AUTOMATION}

$\mathrm{AI}$ is used in marketing automation to engage customers, analyzing their behavior and deliver tailored content to move them through the sales cycle, whether by recommending content, engaging them across different platforms or tailoring email campaigns.

\section{DYNAMIC PRICING}

AI can help brands make more competitive by enabling dynamic pricing. AI platforms can suggest optimal prices for products in real time by evaluating huge quantities of historical and competitive data. It allows brands to adjust prices to reflect demand for certain products, boost sales and edge out of competition.

\section{C) RPA IN SALES AND MARKETING:}

RPA refers to robotic process automation which combines software robots with business defined rules to automate tasks on behalf of an employee.

RPA can be used in sales and marketing departments for various purposes.[4]

\section{COMPETITIVE PRICING AND MONITORING}

RPA solutions for sales and marketing departments are developed so that to keep an eye on competitor's websites and prices of their products in real time. We can get a report or email notification when there is a change and then adjust the prices based on real-time market conditions.

This helps to maintain the competitive edge without taking valuable time from the day to manually browse competitor's website. Let a bot do the work which saves the time for other tasks of sales and marketing departments.

\section{BUSINESS INTELLIGENCE REPORTING}

RPA can be used in business intelligence reporting such as identifying new entrants in the market, notify the new competitor website, track events, campaigns and messaging that our competitor is using to promote products or run sales, providing insights into existing account activity.

\section{DATA AGGRegation And MANAGEMENT}

Robots can be deployed to aggregate contacts for sales staff so that your sales reps do not have to spend time searching for companies, names, phone numbers, and other critical contact information. Bots can be used to update your CRM with this new contact data. Bots can also be used to crawl the web and other software programs, aggregating additional information on your existing CRM contacts and layering additional context onto prospect profiles.

\section{MONITORING RFP /BID SITES FOR NEW OPPORTUNITIES}

RPA can be used to crawl RFP and bid sites for new opportunities. Instead of manually searching for new business, your sales staff can receive new RFPs automatically once they become available. Custom filters may even be developed to weed out projects that aren't the right fit and escalate high- 
value opportunities to key decision makers. Once RFPs have been identified by the bot, RPA can automatically download those files directly in a designated folder on your company's servers or delivered directly to your sales team via email.

\section{[4] AI TOOLS:}

Some of the AI tools used for increasing sales and marketing are [6]:

\section{SALESFORCE}

One of the top players in AI for sales is (not unexpectedly) Salesforce. The company's AI is called "Einstein," and it shows up in many places throughout Salesforce's Customer Success Platform.

Einstein automatically prioritizes leads for your sales reps. It evaluates the likelihood of deals to close. And it empowers developers to bake AI into their Salesforce apps.

Salesforce Einstein is a prime example of how many players in the AI for sales space are existing companies. Big existing players, at least the savvy ones, are incorporating AI into existing platforms by hiring AI talent or buying AI companies.

\section{DRIFT}

Drift helps businesses use conversations to remove friction from their buying process with chat, email, video, and automation products. These products, powered by machine learning, work together to qualify leads $24 / 7$ — essentially cloning your top sales reps.

\section{CONVERSICA}

Conversica is another major player in the space, with $\$ 87$ million in funding. The company provides an automated AI sales assistant that engages your leads in conversation. The AI assistant conducts conversations with leads, further qualifying them before they talk to a rep.

This type of intelligent automation provides a number of benefits. Human reps are freed up to actually sell to people who are interested in a product or service, rather than wasting a lot of time talking to people who aren't qualified. Every lead gets a follow-up, since the AI assistant can scale easily.

It's a perfect example of how machine and humans can collaborate in sales to unlock even more value.

\section{EXCESS.AI}

Exceed.ai uses AI to engage with every sales lead that enters your pipeline, using human-like, two-way conversations by email and chat.

\section{CRAYON}

Crayon's AI-powered competitive intelligence tool tracks 100+ data types across hundreds of millions of sources to tell you exactly what competitors are up to online. This information is then used to automatically build and update sales battlecards that get results. 


\section{[5] CONCLUSION}

Artificial Intelligence has already made its way to various aspects of our life without even letting us realize that. Today AI is used in various areas of business world. AI, Machine learning and robotics have impacted the field of sales and marketing and this impact will magnify significantly in the near future. The marketing field is changing rapidly with the advancement of AI, Machine learning and robotics. The major benefits according to marketing professionals in integrating AI in marketing are increasing efficiency, time-saving in the marketing functions, improving conversion rates, a better understanding of customer information, making marketing decisions more feasible, increasing the ROI, insights, enhanced service and customer satisfaction. Other benefits include improved data analysis and effective handling of marketing processes. With big data, AI and machine learning, sales and marketing will be individualized.

On one hand it is believed that AI will replace sales and marketing professionals, however though the jobs of sales people will get removed, new jobs may be generated as well. The sales and marketing teams need to train their employees for using highly professional AI tools. The need to constantly develop and change their sales and marketing strategies with the development of AI. 


\section{REFERENCES}

[1] https://en.wikipedia.org/wiki/Machine learning - Source (Wikipedia)

[2] https://searchenterpriseai.techtarget.com/definition/AI-Artificial-Intelligence - By Ed Burns, Executive editor, Tech Target

[3] https://www.artificial-solutions.com/blog/homage-to-john-mccarthy-the-father-of-artificialintelligence - By Andy pert, October 29, 2020https://instreamllc.com/how-sales-marketingcan-benefit-from-robotic-process-automationrpa/\#: :text=Robots\%20can\%20be\%20deployed\%20to,with\%20this\%20new\%20contact\%20d ata - Publicly Available Information.

[4] impact of artificial intelligence in marketing and machine learning.pdf-By Muhammad Zafeer Shahid \& Gang Li, Global journals, published in 2019

[5] https://www.marketingaiinstitute.com/blog/ai-in-sales- By Mike kaput, October 8, 2021

[6] https://www.inc.com/adam-fridman/10-examples-of-ai-in-marketing.html- By Adam Fridman, Founder, Inc.

[7] https://www.marketingevolution.com/marketing-essentials/ai-markeitng- Marketing Evolution

[8] https://www.prnewswire.com/news-releases/85-of-marketers-using-artificial-intelligencebelieve-it-will-drive-double-digit-revenue-growth-within-two-years-300823481.html- Source Demandbase , April 03, 2019.

\section{Author[s] brief Introduction}

1. Reshma Motwani - Student of MCA-II (Sem- III), Bharati Vidyapeeth (Deemed to be University) Institute of Management Kolhapur. Mail: motwaniresh16@gmail.com

2. Mayuri Patel - Student of MCA-II (Sem- III), Bharati Vidyapeeth (Deemed to be University) Institute of Management Kolhapur. Mail: mayuripate14727@gmail.com

3. Dr. Shabana Memon - Assistant Professor, Dept. of Management Studies, Bharati Vidyapeeth (Deemed to be University) Institute of Management Kolhapur. Mail:

shabana.memon@bharatividyapeeth.edu

4. Prof. Nripesh Nrip - Assistant Professor, Dept. of Computer Application, Bharati Vidyapeeth (Deemed to be University) Institute of Management Kolhapur. 